\title{
EXTREMAL THEORY OF ORDERED GRAPHS
}

\author{
GÁBOR TARDOS
}

\begin{abstract}
We call simple graphs with a linear order on the vertices ordered graphs. Turántype extremal graph theory naturally extends to ordered graphs. This is a survey on the ongoing research in the extremal theory of ordered graphs with an emphasis on open problems.
\end{abstract}

\section{Definitions}

An ordered graph is a simple graph with linear order on the vertices. Formally, an ordered graph is triple $(V, E,<)$, where $V$ is the vertex set, $E \subseteq\left(\begin{array}{c}V \\ 2\end{array}\right)$ is the edge set and $<$ is a linear order relation on $V$. In this survey we assume that $V$ is finite. We say that $(V, E)$ is the simple graph underlying the ordered graph $(V, E,<)$ and that the ordered graph $(V, E,<$ ) is an ordering of the simple graph $(V, E)$. The notion of subgraph and isomorphism naturally extend to ordered graphs: the ordered graphs $(V, E,<)$ and $\left(V^{\prime}, E^{\prime},<^{\prime}\right)$ are isomorphic if there is an order preserving isomorphism between the graphs $(V, E)$ and $\left(V^{\prime}, E^{\prime}\right)$. The ordered graph $\left(V^{\prime}, E^{\prime},<^{\prime}\right)$ is an ordered subgraph of $(V, E,<)$ if $V^{\prime} \subseteq V$, $E^{\prime} \subseteq E$ and $<^{\prime}$ is the restriction of $<$ to $V^{\prime}$.

Armed with this definition we can extend some classic areas of graph theory to ordered graphs. Here we do this for Turán-type extremal graph theory. It asks for the maximal number of edges in a simple graph of given size that avoids (i.e., does not contain as a subgraph) a specified pattern or all members of a given family of patterns. In particular, we are interested in the maximal number, $\operatorname{ex}(\mathbb{P}, n)$, of edges in an $n$-vertex simple graph that has no subgraph isomorphic to any member of the family $\mathbb{P}$. Note that we must require that $P$ does not contain empty graphs in order for this definition to make sense. If the forbidden pattern is a singleton we write $\operatorname{ex}(P, n)$ to denote $\operatorname{ex}(\{P\}, n)$. We call $\operatorname{ex}(\mathbb{P}, n)$ the extremal function of the family $\mathbb{P}$ and will concentrate on its asymptotic

Supported by the Cryptography "Lendület" project of the Hungarian Academy of Sciences and by the National Research, Development and Innovation Office, NKFIH projects K-116769 and SNN-117879.

MSC2010: 05C35. 
behavior. Accordingly, all the asymptotic notations like $O(\cdot), o(\cdot)$ should be interpreted for a fixed family $\mathbb{P}$ and, in particular, the implied constants in $O(\cdot)$ may depend on this family.

For a natural extension of this theory to ordered graphs, we consider a family $\mathbb{P}$ of ordered graphs and we are looking for the largest number $\operatorname{ex}_{<}(\mathbb{P}, n)$ of edges in an $n$-vertex ordered graph with no ordered subgraph isomorphic to any member of $\mathbb{P}$. As before, we require that each member of $P$ has at least one edge and simplify the notation for singleton families by writing $\operatorname{ex}_{<}(P, n)$ to denote $\operatorname{ex}_{<}(\{P\}, n)$. Our remark on the asymptotic notation also applies here.

Let us first observe that the extremal theory of ordered graph is strictly richer than classical extremal graph theory in the sense that the classical questions can be equivalently asked in this setting, but we can also ask new questions. In particular, for any family $P$ of simple graphs one can form the family $P_{<}$consisting all orderings of the patterns in $P$ and then we trivially have:

$$
\operatorname{ex}(\mathbb{P}, n)=\operatorname{ex}_{<}\left(P_{<}, n\right) .
$$

On the other hand, if we forbid, say, a single ordered graph $P$, the corresponding extremal function $\operatorname{ex}_{<}(P, n)$ has no direct analogue in the classical theory. We naturally have $\operatorname{ex}_{<}(P, n) \geq \operatorname{ex}(\bar{P}, n)$, where $\bar{P}$ is the simple graph underlying $P$, but this lower bound is typically very weak, since avoiding $\bar{P}$ in a particular order is often much easier than avoiding it in all possible orders.

Extensions of Ramsey theory to ordered graph is also studied extensively, see Balko, Cibulka, Král, and Kynčl [2015] and Conlon, Fox, Lee, and Sudakov [2017].

\section{Basic results}

Any survey about extremal graph theory should start with the following classical theorem of Turán [1941], of which the $r=2$ special case (the maximal number of edges in a triangle-free graph) was proved by Mantel in 1907. The result gives the exact extremal function when the forbidden graph is a complete graph. Further, for the $(r+1)$-vertex complete graph $K_{r+1}$ the theorem states that the unique (up to isomorphism) $n$-vertex graph with the maximum number of edges avoiding $K_{r+1}$ is the Turán graph $T(n, r)$ formed by partitioning the vertices into $r$ almost equal parts and letting a pair of vertices form an edge if and only if they are from distinct parts. Note that the number of edges of the Turán graph $T(n, r)$ is $(1-1 / r) n^{2} / 2-O(1)$, where the $O(1)$ error term comes from unequal parts and can go as high as $\lfloor r / 8\rfloor$. As a consequence, we have:

Theorem 1 (Turán [ibid.]). For every $r \geq 1$ we have

$$
\operatorname{ex}\left(K_{r+1}, n\right)=\left(1-\frac{1}{r}\right) \frac{n^{2}}{2}-O(1)
$$


A trivial generalization of this result to ordered graphs involves the ordered clique, the unique ordering of the complete graph. Let $K_{r+1,<}$ stand for the $(r+1)$-vertex ordered clique and we trivially have $\operatorname{ex}_{<}\left(K_{r+1,<}, n\right)=\operatorname{ex}\left(K_{r+1}, n\right)$. A more revealing generalization is about the ordered path $P_{r+1,<}$ obtained from the $(r+1)$-vertex path $P_{r+1}$ with the natural order on the vertices where edges connect neighboring vertices in the order. We have $\operatorname{ex}_{<}\left(P_{r+1,<}, n\right)=\operatorname{ex}\left(K_{r+1}, n\right)$. Here the direction $\leq$ follows from the fact that $P_{r+1,<}$ is an ordered subgraph of $K_{r+1,<}$ and $\geq$ follows from the fact that if we order the vertices of $T(n, r)$ in a way that the $r$ parts become intervals in the ordering, then the resulting ordered graph does not contain $P_{r+1,<}$ as an ordered subgraph. Note, however, that in the case $r$ does not divide $n$, this process may yield several non-isomorphic extremal ordered graphs. Note also that the path $P_{r+1}$ has several non-isomorphic orderings for $r>1$, and by Theorem 3 below, all other orderings have smaller extremal functions.

The most general result in Turán-type extremal graph theory is the following consequence of the Erdős-Stone theorem, Erdős and Simonovits [1966]. It basically states that the extremal function of any simple graph is close to the extremal function of the complete graph with the same chromatic number.

Theorem 2 (Erdős and Stone [1946] and Erdős and Simonovits [1966]). Let $\mathbb{P}$ be a family of simple graphs and $r+1=\min _{P \in \mathbb{P}} \chi(P)$ be the smallest chromatic number of a member of this family. We have

$$
\operatorname{ex}(\mathbb{P}, n)=\left(1-\frac{1}{r}\right) \frac{n^{2}}{2}+o\left(n^{2}\right)
$$

Pach and Tardos [2006] gave a generalization of this result for ordered graphs. It is based on finding the "correct" version of the chromatic number for ordered graph.

The interval coloring of an ordered graph is a proper coloring of the underlying simple graph in which each color class is an interval of the linear order. The interval chromatic number of an ordered graph $P$ is the smallest number of colors in an interval coloring of $P$. We write $\chi<(P)$ to denote the interval chromatic number of $P$.

Note that the interval chromatic number is much simpler to compute than the chromatic number because a greedy strategy suffices. Indeed, we can form the first color class by taking longest initial segment of the vertices that form an independent set and proceed similarly for subsequent color classes. The process yields an interval coloring with the fewest possible colors. Using this definition, the generalization of the Erdös-Stone-Simonovits theorem is rather straightforward:

Theorem 3 (Erdős-Stone-Simonovits Theorem for ordered graphs Pach and Tardos [ibid.]). Let $\mathbb{P}$ be a family of ordered graphs and $r+1=\min _{P \in \mathbb{P}} \chi_{<}(P)$ be the smallest interval chromatic number of a member of this family. We have

$$
\operatorname{ex}_{<}(\mathbb{P}, n)=\left(1-\frac{1}{r}\right) \frac{n^{2}}{2}+o\left(n^{2}\right) .
$$


Just as the classic version of this theorem, it gives exact asymptotics for the extremal function of ordered graphs unless the ordered graph is ordered bipartite (i.e., has interval chromatic number 2). We will therefore concentrate on ordered bipartite graphs. Containment between ordered bipartite graphs can also be visualized using the language of containment in 0-1 matrices. This connection is explored in the next section.

\section{Connection to 0-1 matrices}

A $0-1$ matrix is simply a matrix with all entries being 0 or 1 . The weight of such a matrix is the number of its 1 -entries. A $0-1$ matrix $A$ is said to contain another $0-1$ matrix $P$ if $P$ is a submatrix of $A$ or $P$ is obtained from a submatrix of $A$ by replacing some 1entries with 0 -entries. Note that permuting rows or columns is not allowed. If $A$ does not contain $P$, we say it avoids $P$. The extremal problem for $0-1$ matrix containment can be formulated as computing (or estimating) the following extremal function for families $P$ of 0-1 matrices: $\operatorname{Ex}(P, n)$ is the maximal weight of an $n$-by- $n$ 0-1 matrix that avoids all matrices in $P$. We require that all matrices in $P$ have positive weights. We write $\operatorname{Ex}(P, n)$ to denote $\operatorname{Ex}(\{P\}, n)$.

For a 0 -1 matrix $P$, let $G_{P}$ stand for the ordered bipartite graph whose vertices correspond to the rows and columns of $P$, the order of the vertices agrees with the order of rows and columns in $P$ with all row-vertices preceding all column vertices, and with an edge between a row-vertex and a column-vertex if and only if the corresponding entry in $P$ is 1. This makes $P$ the bipartite adjacency matrix of $G_{P}$ and turns the weight of $P$ into into the number of edges in $G_{P}$. The close connection between the extremal theory of ordered bipartite graphs and 0-1 matrices follows from the trivial observation that if a $0-1$ matrix $A$ contains another 0-1 matrix $P$, then the ordered graph $G_{A}$ also contains $G_{P}$. The converse is also true if the homomorphism of $G_{P}$ to $G_{A}$ maps row-vertices to row-vertices and column-vertices to column-vertices. This extra condition is automatically satisfied if both the last row and first column of $P$ contain at least one 1-entry, so in this case we have $\operatorname{Ex}(P, n) \leq \operatorname{ex}_{<}\left(G_{P}, 2 n\right)$. There is no equality in general, because $\operatorname{ex}_{<}\left(G_{P}, 2 n\right)$ is the maximum number of edges among all ordered graphs on $2 n$ vertices avoiding $G_{P}$ and the extremal ones may not be ordered bipartite. Still, the two extremal functions are really close to each other as shown by the following observation:

Theorem 4 (Pach and Tardos [2006]). For a 0-1 matrix $P$ and the corresponding ordered bipartite graph $G_{p}$ we have

$$
\operatorname{Ex}(n, P) \leq \operatorname{ex}_{<}\left(2 n, G_{p}\right)=O(\operatorname{Ex}(n, P) \log n) .
$$


The logarithmic term in the bound above is needed even for some small matrices, e.g., for the matrix

$$
P=\left(\begin{array}{ll}
1 & 1 \\
0 & 1
\end{array}\right) .
$$

For this matrix, we have $\operatorname{Ex}(n, P)=2 n-1$, but for the corresponding ordered graph $G_{P}$ one has $\operatorname{ex}_{<}\left(n, G_{P}\right)=n \log n+O(n)$, where log stands for the binary logarithm. A construction showing the lower bound for this estimate is an ordered graph whose vertices are adjacent if and only if their distance in the ordering is a power of 2.

The extremal theory of $0-1$ matrices predates the related theory of ordered graphs. Füredi [1990] established the extremal function for a specific 2-by-3 0-1 matrix and used this result for a problem in combinatorial geometry: he bounded the number of diagonals of equal length in a convex $n$-gon. Independently, Bienstock and Gyorri [1991] found the extremal function of few small 0-1 matrices. Later Füredi and Hajnal [1992] started a systematic study of the extremal theory of $0-1$ matrices. This latter paper not only contained many nice results, but was also rich in conjectures and had a significant effect on future research. As we will see, some of these conjectures have since been proved, others disproved and some are still open.

\section{Relation between ordered and unordered extremal functions}

A (too) general conjecture that appeared in Füredi and Hajnal [ibid.] can be informally stated as

Conjecture 1. For all 0-1 matrix $P$ of positive weight we have

$$
\operatorname{Ex}(P, n) \approx \operatorname{ex}\left(\bar{G}_{P}, n\right),
$$

where $\bar{G}_{P}$ is the simple graph underlying the ordered graph $G_{P}$.

This conjecture connects ordered extremal theory to the classical unordered one. We clearly have an inequality in one direction:

$$
\operatorname{Ex}(n, P) \leq \operatorname{ex}_{<}\left(2 n, G_{P}\right) \leq \operatorname{ex}\left(2 n, \bar{G}_{P}\right)=O\left(\operatorname{ex}\left(n, \bar{G}_{P}\right)\right) .
$$

By Theorem 4, the first inequality is almost tight for any pattern, so we concentrate of the second inequality and ask how large the ratio between the two sides can be:

Question 1. How high can the ratio $\frac{\mathrm{ex}<(n, P)}{\operatorname{ex}(n, \bar{P})}$ be for an ordered bipartite graph $P$ with more than two vertices and at least one edge and its underlying simple graph $\bar{P}$ ?

The paper Pach and Tardos [2006] gives an ordering $P_{k}$ of the cycle $C_{2 k}$ with $\operatorname{ex}_{<}\left(n, P_{k}\right)=$ $\Omega\left(n^{4 / 3}\right)$. Using the Bondy-Simonovits theorem on the extremal function of cycles Bondy 
and Simonovits [1974], one obtains that the ratio in Question 1 for the pattern $P=P_{k}$ is $\Omega\left(n^{1 / 3-1 / k}\right)$, which disproves Conjecture 1 . We do not know if any pattern with higher ratio, say $\Omega\left(n^{1 / 3}\right)$ exists. For an upper bound, we trivially have $O(n)$, as both the enumerator and the denominator are functions between $n$ and $n^{2}$. In fact, they are $O\left(n^{2-\epsilon}\right)$ for some $\epsilon>0$ depending on the size of $P$ by the Kövári-Sós-Turán theorem Kövari, Sós, and Turán [1954], so the ratio is always $O\left(n^{1-\epsilon}\right)$, but no better upper bound is known.

\section{Forests}

The Füredi and Hajnal [1992] formulated the special case of Conjecture 1 for cycle-free patterns $P$ separately. Here we call a $0-1$ matrix $P$ cycle-free if the corresponding simple graph $\bar{G}_{P}$ is cycle-free, that is a forest. In this case, $\operatorname{ex}\left(n, \bar{G}_{P}\right)$ (the extremal function of an unordered forest) is trivially linear. Concerning the corresponding question for ordered graphs, we formulate the following conjecture:

Conjecture 2 For an ordered bipartite forest $P$ and any $c>1$, we have

$$
\operatorname{ex}_{<}(P, n)=o\left(n^{c}\right) .
$$

Note first that if this conjecture is true, then it characterizes the ordered graphs with almost linear extremal functions. Indeed, if $P$ is not ordered bipartite, then $\operatorname{ex}_{<}(P, n)=$ $\Theta\left(n^{2}\right)$ by Theorem 3 , while if the underlying graph $\bar{P}$ contains a cycle, then $\operatorname{ex}_{<}(P, n) \geq$ $\operatorname{ex}(\bar{P}, n)=\Omega\left(n^{c}\right)$ for some $c>1$.

Note that $o\left(n^{c}\right)$ for all $c>1$ is not the only possible way to quantify the notion that a function is "close to linear". One could formulate a stronger conjecture with a bound $O\left(n \log ^{c} n\right)$ for a constant $c=c_{P}$ depending on $P$, or even with an $O(n \log n)$ bound. Conjecture 2 and the conjecture with the $O\left(n \log ^{c} n\right)$ bound are still open and by Theorem 4 are equivalent to the similar conjectures about $\operatorname{Ex}(P, n)$ for cycle-free 0 - 1 matrices $P$. The strongest form of the conjecture (an $O(n \log n)$ bound) was also considered for a while and was supported by the fact that it was easy to find an extremal function of the order $\Theta(n \log n)$, but there was no known example of an ordered bipartite forest whose extremal function grows faster. Note that here the distinction between cycle-free 0-1 matrices and ordered bipartite forests is meaningful. As we have seen above, there exists a three-edge ordered bipartite path whose extremal function is $\Theta(n \log n)$. Although the extremal function of the corresponding 2-by-2 matrix is linear, there is a 3-by-2 0-1 matrix whose extremal function is $\Theta(n \log n)$. This was the first $0-1$ matrix considered in the context of extremal functions in the papers Füredi [1990] and Bienstock and Györi [1991].

Pettie [2011] found a cycle-free 0-1 matrix $P$ with extremal function slightly higher than $n \log n$ : for this matrix $P$ one has $\operatorname{Ex}(P, n)=\Omega(n \log n \log \log n)$. By this, he 
disproved the strengthening of Conjecture 2 with the $O(n \log n)$ upper bound, but the conjecture may still hold with the bound $O\left(n \log ^{2} n\right)$. Pettie's result was slightly improved and the current best lower bound is due Park and Shi [n.d.]. They found a cycle-free 0-1 matrix $P_{m}$ with $\operatorname{Ex}\left(P_{m}, n\right)=\Omega\left(n \log n \log \log n \cdots \log { }^{(m)} n\right)$, where $\log { }^{(m)}$ denotes the $m$-times-iterated logarithm function.

On the positive side, $\operatorname{ex}_{<}(P, n)=O\left(n \log ^{c} n\right)$ was established in Pach and Tardos [2006] for all ordered bipartite forests with at most 6 vertices. The most general result in this direction is due to Korándi, Tardos, Tomon, and Weidert [2017]. They call a 0-1 matrix $M$ vertically degenerate if for any submatrix $M^{\prime}=\left(a_{i j}\right)$ of $M$ consisting of $l>1$ rows one can find $1 \leq k<l$ such that $M^{\prime}$ has at most one column $j$ with two 1 -entries $a_{i j}=a_{i^{\prime} j}=1$ satisfying $1 \leq i \leq k<i^{\prime} \leq l$. Note that all vertically degenerate $0-1$ matrices are cycle-free. All cycle-free $0-1$ matrices with at most three rows are vertically degenerate, but there are 4-row cycle-free 0-1 matrices that are not vertically degenerate. Using a density increment argument they prove the following theorem.

Theorem 5 (Korándi, Tardos, Tomon, and Weidert [ibid.]). Let $M$ be a vertically degenerate 0-1 matrix with l rows. We have

$$
\mathrm{Ex}_{<}(M, n)=n 2^{O\left(\log ^{1-1 / l} n\right)} .
$$

This result implies that Conjecture 2 holds for all ordered graphs $G_{M}$, where $M$ is a vertically degenerate $0-1$ matrix. By symmetry, Conjecture 2 is also true for all $G_{M}$, where $M$ is horizontally degenerate, that is, the transpose of $M$ is vertically degenerate. Conjecture 2 has not been verified for any other ordered bipartite forest. The smallest of these open cases is an ordered path on 8 vertices.

\section{Linear extremal functions}

Füredi and Hajnal [1992] conjectured, and later Marcus and Tardos [2004] proved, that $\operatorname{Ex}(P, n)=O(n)$ for permutation matrices $P$. It is not hard to see that this result can be restated in the following equivalent form (although Theorem 4 does not directly imply this equivalence).

Theorem 6. The extremal function of any ordered bipartite matching $P$ is linear. That is,

$$
\operatorname{Ex}(P, n)=O(n) .
$$

Conjecture 2, if true, characterizes all ordered graphs with almost linear extremal functions. It would be nice to find a characterization of ordered graphs or 0-1 matrices with linear extremal functions. One possibility is finding all minimally nonlinear matrices. We call a 0-1 matrix $P$ minimally nonlinear, if its extremal function $\operatorname{Ex}(P, n)$ is nonlinear, 
but $\operatorname{Ex}\left(P^{\prime}, n\right)=O(n)$ for all 0 -1 matrices $P^{\prime} \neq P$ contained in $P$. It might be possible to find such a characterization, but the following theorem indicates that this might be a difficult task:

Theorem 7 (Geneson [2009] and Keszegh [2009]). There are infinitely many minimally nonlinear matrices.

\section{Interaction between ordered graphs}

We finish this survey with a few remarks on interactions between extremal functions of different forbidden patterns. Let us start with the classical extremal theory of graphs. Clearly, we have

$$
\operatorname{ex}(\{G, H\}, n) \leq \min (\operatorname{ex}(G, n), \operatorname{ex}(H, n)) .
$$

By Theorem 2, the two sides are asymptotically the same for non-bipartite graphs $G$ and $H$. It is easy to see that they differ by a factor of less than 2 if only one of the graphs is bipartite. For bipartite graphs, the situation is more complicated. We say that $G$ and $H$ interact if the two sides differ more than by a constant factor. It is not known if there exists any interacting pair of graphs, but Faudree and Simonovits [n.d.] conjecture that the cycle $C_{4}$ and the subdivision of the complete graph $K_{4}$, in which each edge is subdivided with a single new vertex, do interact.

In contrast, for 0-1 matrices it is not hard to find a lot of interactions. Consider the 3-by-2 matrix $M_{1}=\left(\begin{array}{lll}1 & 1 & 0 \\ 1 & 0 & 1\end{array}\right)$. Füredi [1990] and Bienstock and Györi [1991] proved that $\operatorname{Ex}\left(M_{1}\right)=\Theta(n \log n)$. By symmetry, the extremal functions of the matrices $M_{2}=\left(\begin{array}{lll}1 & 0 & 1 \\ 1 & 1 & 0\end{array}\right)$, $M_{3}=\left(\begin{array}{lll}0 & 1 & 1 \\ 1 & 0 & 1\end{array}\right)$ and $M_{4}=\left(\begin{array}{lll}1 & 0 & 1 \\ 0 & 1 & 1\end{array}\right)$ are same. The following theorem implies that each of $M_{2}$, $M_{3}$ and $M_{4}$ interacts with $M_{1}$ :

Theorem 8 (Tardos [2005]).

$$
\begin{gathered}
\operatorname{Ex}\left(\left\{M_{1}, M_{2}\right\}, n\right)=\Theta(n) \\
\operatorname{Ex}\left(\left\{M_{1}, M_{3}\right\}, n\right)=\Theta(n \log n / \log \log n) \\
\operatorname{Ex}\left(\left\{M_{1}, M_{4}\right\}, n\right)=\Theta(n \log \log n)
\end{gathered}
$$

These results represent the first step toward exploring interactions between different patterns. It would be interesting to find "stronger" interactions, where the ratio between the right and left sides of $(*)$ is larger than logarithmic.

Question 2 Are there ordered graphs $G$ and $H$ such that

$$
\operatorname{ex}_{<}(\{G, H\}, n)=O\left(\min \left(\operatorname{ex}_{<}(G, n), \operatorname{ex}_{<}(H, n)\right) / n^{\epsilon}\right)
$$

for some $\epsilon>0$ ? 


\section{References}

Martin Balko, Josef Cibulka, Karel Král, and Jan Kynčl (2015). "Ramsey numbers of ordered graphs". Electronic Notes in Discrete Mathematics 49, pp. 419-424 (cit. on p. 3254).

Dan Bienstock and Ervin Győri (1991). "An extremal problem on sparse 0-1 matrices". SIAM J. Discrete Math. 4.1, pp. 17-27. MR: 1090285 (cit. on pp. 3257, 3258, 3260).

J. A. Bondy and M. Simonovits (1974). "Cycles of even length in graphs". J. Combinatorial Theory Ser. B 16, pp. 97-105. MR: 0340095 (cit. on p. 3257).

David Conlon, Jacob Fox, Choongbum Lee, and Benny Sudakov (2017). "Ordered Ramsey numbers”. J. Combin. Theory Ser. B 122, pp. 353-383. MR: 3575208 (cit. on p. 3254).

P. Erdős and M. Simonovits (1966). "A limit theorem in graph theory”. Studia Sci. Math. Hungar 1, pp. 51-57. MR: 0205876 (cit. on p. 3255).

P. Erdős and A. H. Stone (1946). "On the structure of linear graphs". Bull. Amer. Math. Soc. 52, pp. 1087-1091. MR: 0018807 (cit. on p. 3255).

R. J. Faudree and M. Simonovits (n.d.). "On a class of degenerate extremal graph problems II" (cit. on p. 3260).

Zoltán Füredi (1990). "The maximum number of unit distances in a convex $n$-gon”. $J$. Combin. Theory Ser. A 55.2, pp. 316-320. MR: 1075714 (cit. on pp. 3257, 3258, 3260). Zoltán Füredi and Péter Hajnal (1992). "Davenport-Schinzel theory of matrices". Discrete Math. 103.3, pp. 233-251. MR: 1171777 (cit. on pp. 3257-3259).

Jesse T. Geneson (2009). "Extremal functions of forbidden double permutation matrices". J. Combin. Theory Ser. A 116.7, pp. 1235-1244. MR: 2527608 (cit. on p. 3260).

Balázs Keszegh (2009). “On linear forbidden submatrices”. J. Combin. Theory Ser. A 116.1, pp. 232-241. MR: 2469261 (cit. on p. 3260).

Dániel Korándi, Gábor Tardos, István Tomon, and Craig Weidert (Nov. 2017). “On the Turán number of ordered forests". arXiv: 1711.07723 (cit. on p. 3259).

T. Kövari, V. T. Sós, and P. Turán (1954). “On a problem of K. Zarankiewicz”. Colloquium Math. 3, pp. 50-57. MR: 0065617 (cit. on p. 3258).

Adam Marcus and Gábor Tardos (2004). "Excluded permutation matrices and the StanleyWilf conjecture". J. Combin. Theory Ser. A 107.1, pp. 153-160. MR: 2063960 (cit. on p. 3259).

János Pach and Gábor Tardos (2006). "Forbidden paths and cycles in ordered graphs and matrices". Israel J. Math. 155, pp. 359-380. MR: 2269435 (cit. on pp. 3255-3257, 3259).

S. G. Park and Q. Shi (n.d.). "New bounds on extremal numbers in acyclic ordered graphs" (cit. on p. 3259). 
Seth Pettie (2011). "Degrees of nonlinearity in forbidden 0-1 matrix problems". Discrete Math. 311.21, pp. 2396-2410. MR: 2832138 (cit. on p. 3258).

Gábor Tardos (2005). "On 0-1 matrices and small excluded submatrices”. J. Combin. Theory Ser. A 111.2, pp. 266-288. MR: 2156213 (cit. on p. 3260).

P. Turán (1941). “On an extremal problem in graph theory”. Matematikai és Fizikai Lapok 48, pp. 436-452 (cit. on p. 3254).

Received 2018-03-01.

GÁBOR TARDOS

RÉNyi InSTITUTE of Mathematics

BUDAPEST

HunGaRY

tardos@renyi.hu

tardosgabor@gmail.com 